\title{
Measurement of knee cartilage thickness in young adults by musculoskeletal ultrasound in relation to serum vitamin $D$ level
}

\author{
Ashraf I. Khalifa', Mohamed M. Abdelkreem ${ }^{1}$, Mohamed A. Hashesh ${ }^{2}$, Khaled O. Zaiton ${ }^{3}$ \\ ${ }^{1}$ Department of Physical medicine \& Rheumatology and Rehabilitation, \\ ${ }^{2}$ Department of clinical pathology, Faculty of Medicine, Al-Azhar University, Cairo, \\ ${ }^{3}$ Department of Physical medicine \& Rheumatology and Rehabilitation, AL-Ahrar educational hospital, Zagazig, Egypt \\ Corresponding author: Khaled O. Zaiton; Mobile: 01000089394; Email: ziton8080@gmail.com
}

\begin{abstract}
Background: Osteoarthritis (OA) is a degenerative joint disease involving the cartilage and many of it's surrounding tissues. In addition to damage and loss of articular cartilage, there is remodeling of subarticular bone, osteophyte formation, ligamentous laxity, weakening of periarticular muscles and in some cases, synovial inflammation.Musculoskeletal Ultrasound (MSUS) is a newer imaging technique in musculoskeletal system for diagnosis and monitoring of treatment. A potential association between serum vitamin D levels and knee OA symptoms may occur through effect on cartilage metabolism, bone metabolism or both.

Aim of the Work: This study was done tomeasure of knee cartilage thickness in young adults by musculoskeletal ultrasound in relation to serum $25(\mathrm{OH})$ vitamin D level.

Patients and Methods: This study was carried on 75 individuals chosen from out-patient's clinics of physical medicine and rheumatology department at AL-Hussein hospitals of AL-Azhar University after their agreement to inform a consent for this research. They are divided into 2 groups: Group (1) includes 50 patients suffering

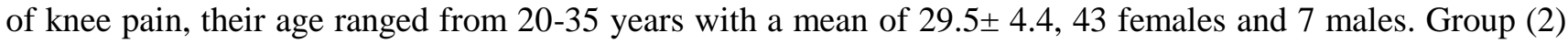
includes 25 apparent healthy volunteers matched for age and sex as group 1.

Results: Musculoskeletal Ultrasonography is a very important tool in detection of early OA in patients with vitamin D deficiency and Comparison between MRI and musculoskeletal ultrasound showed high concordance in the findings of cartilage changes of early OA patients.

Conclusion: Vitamin D deficiency and insufficiency is correlated with thinning of femoral cartilage thickness and higher risk of early knee OA.
\end{abstract}

Keywords: knee cartilage thickness, musculoskeletal ultrasound, vitamin D, Magnetic Resonance Imaging.

\section{INTRODUCTION}

The concepts regarding OA have dramatically changed in the past decade by development of the new imaging techniques as musculoskeletal ultrasound and MRI these techniques allow detection of early detection of pre-radiographic structural damages and not only the periarticular bone but also in synovial membrane, ligaments, cartilage and menisci ${ }^{(\mathbf{1})}$.

Knee OA is one of the clinical forms of OA with clinical manifestations and functional disorders which can be visualized by X-ray, Computed Tomography (CT), or Magnetic Resonance Imaging (MRI) ${ }^{(2)}$.

These imaging techniques help to evaluate and classify degenerative changes of OA, X-ray is widely used for knee OA classification but does not evaluate soft tissue of the knee $^{(3)}$.

Musculoskeletal Ultrasound (MSUS) is a newer imaging technique in musculoskeletal system for diagnosis and monitoring of treatment. High-frequency and high-resolution US of joints does not assess only morphologic changes but also functional changes in the knee joint. Static and dynamic evaluations are big advantages of this imaging techniques ${ }^{(4)}$.

A potential association between serum vitamin D levels and knee OA symptoms may occur through effect on cartilage metabolism, bone metabolism or both ${ }^{(5)}$.

MRI became popular technique that is more sensitive than to the OA changes including abnormalities in cartilage, subchondral bone, menisci, ligaments and synovium ${ }^{(6)}$.

\section{AIM OF THE WORK}

This study was done to measure of knee cartilage thickness in young adults by musculoskeletal ultrasound in relation to serum $25(\mathrm{OH})$ vitamin D level.

\section{PATIENTS AND METHODS}

This study was carried on 75 individuals chosen from out-patient's clinics of physical medicine and rheumatology department at AL-Hussein hospitals of AL-Azhar University after their agreement to inform a consent for this research. They are divided into 2 groups: Group (1) includes 50 patients suffering of knee pain, their age ranged from 
20-35 years with a mean of $29.5 \pm 4.4,43$ females and 7 males. Group (2) includes 25 apparent healthy volunteers matched for age and sex as group 1 .

Exclusion criteria: Patients with other rheumatic condition such as rheumatoid arthritis. Trauma. Infection. Malignancy. Knee injection with steroid in the last 6 months. Vitamin D supplements. Chronic renal diseases. Thyroid diseases. Chronic GIT and Pulmonary diseases.

Investigations: (1) Clinical evaluation: included full history taking, clinical examination and assessment of knee pain, stiffness, and physical function in group 1 patients were done according to Western Ontario and McMaster Universities Osteoarthritis Index (WOMAC), and musculoskeletal examination. (2) Laboratory evaluation in the form of: $\mathrm{CBC}, \mathrm{ESR}, \mathrm{CRP}$, lipid profile, TSH, serum 25(OH) vitamin D level (3) Radiological assessment by plain $\mathrm{x}$-ray on both knees (sky, lateral, anteroposterior views) and MRI on both knees.

The procedure: Musculoskeletal ultrasound scan was done for all patients and control group. They were evaluated by musculoskeletal ultrasound using a linear probe7-12 MHz. Distal femoral cartilage thickness was assessed while patient lied in supine position with their knees in maximum flexion. The transducer was positioned axially over the patellar outer edge. The distance between the thin hyperechoic line at the synovial space/cartilage interface and the sharp hyperechoic line at the cartilage bone interface was interpreted as the cartilage thickness. Measurements were obtained from the central points of the right medial condyle (RMC), right intercondylar area (RIC), left medial condyle (LMC), left lateral condyle (LLC), and left intercondylar area (LIC).

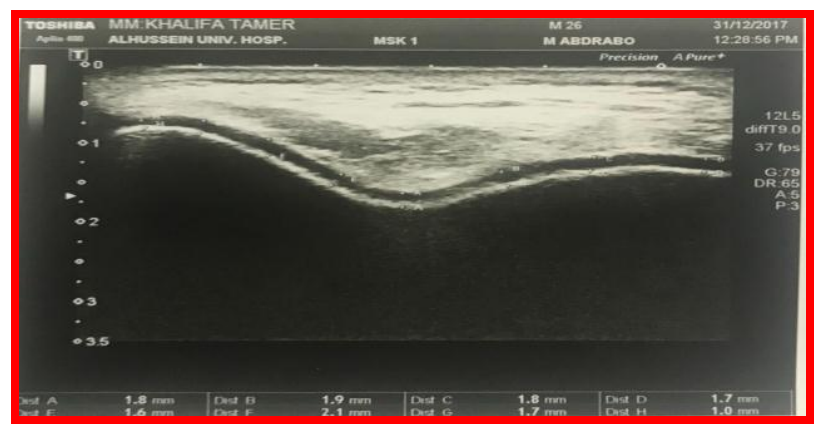

Fig. (1): Ultrasound showing loss of the normal sharpness of cartilage interfaces and local thinning of RT knee cartilage less than $50 \%$ grade $2 \mathrm{~A}$ saarakkala grading scale.

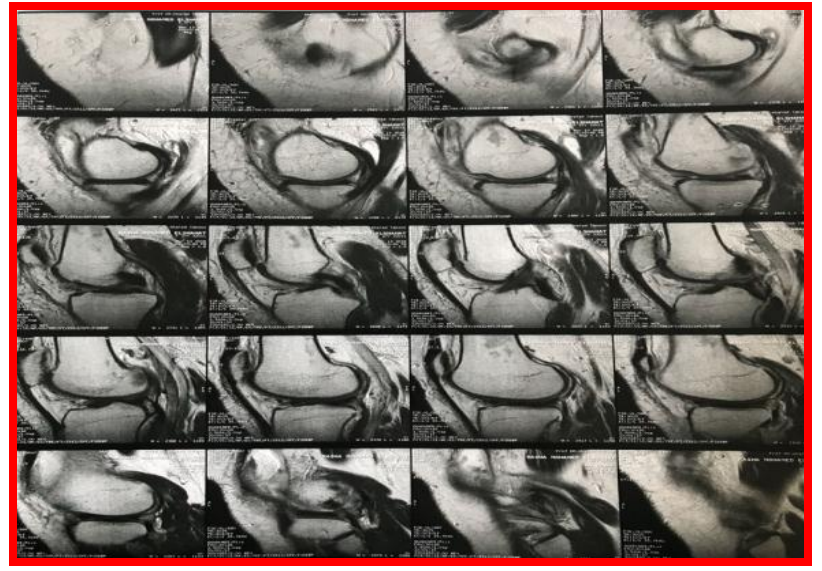

Fig. (2): MRI Showing early osteo-arthritis knee with grade 2 posterior horn medial meniscus degeneration.

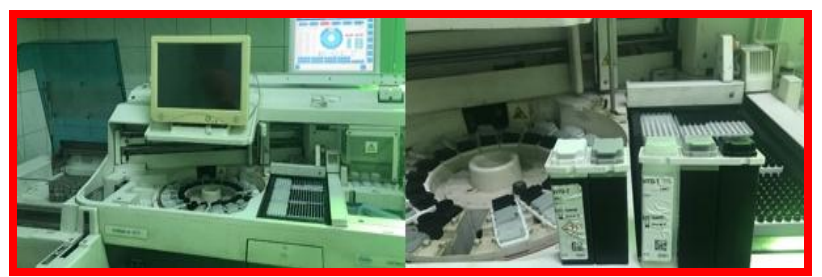

Fig. (3): Shows COBAS 4000 and kits of vitamin D.

\section{Statistical analysis}

Data were analyzed by Statistical Package of Social Science (SPSS) 2016, Continuous variables were presented as the Mean $\pm \mathrm{SD}$ if normally distributed or Median (Range) if not normally distributed. Normality was checked by Shapiro-Wilk test. Homogeneity of variances was checked by Leven's test. Categorical variables were presented by the count and percentage if nominal variables or presented by Median (Range) if ordinal variables.

Independent-samples $\mathbf{t}$-test: is used to determine if a difference 0continuous dependent variable.

Mann-Whitney u test (nonparametric alternative to independent-samples t-test). It is used to determine if a difference exists between the medians of two independent groups on a continuous dependent variable or an ordinal dependent variable.

The chi-squared test: is used to discover if there is a relationship between two categorical variables.

A receiver operating characteristic (ROC) curve: is used to determine the threshold value for optimal sensitivity and specificity of a test. 
Probability (P-value): P-value $<0.05$ was considered significant. P-value $<0.001$ was considered as highly significant. P-value $>0.05$ was considered insignificant.

\section{RESULTS}

Table (1): Dunn's post hoc test for the patient groupregarding ultrasound grading.

\begin{tabular}{|c|c|c|c|}
\hline \multirow{2}{*}{$\begin{array}{c}\text { Ultrasound } \\
\text { grading }\end{array}$} & Deficient & Insufficient & Sufficient \\
\cline { 2 - 4 } & $\mathbf{( < \mathbf { 1 0 } )}$ & $\mathbf{( 1 0 - 3 0 )}$ & $\mathbf{( \geq \mathbf { 3 0 } )}$ \\
\cline { 2 - 4 } & $\mathbf{n = 2 4}$ & $\mathbf{n = 4 5}$ & $\mathbf{n}=\mathbf{3}$ \\
\hline \multicolumn{4}{|c|}{ Median (Range) } \\
\hline & $2(1-2)$ & $2(1-2)$ & $1(1-1)$ \\
\hline Deficient (<10) & & .085 & $.003^{* *}$ \\
\hline Insufficient (10-20) & & & $.04^{*}$ \\
\hline
\end{tabular}

Dunn's post hoc test revealed that patients with deficient and insufficient vitamin D level had statistically significantly worse ultrasound grading compared with patients with sufficient vitamin D level ( $\mathrm{P}<.003, \mathrm{P}=.04$ respectively).

Table (2): Shows degree of agreement between US grading and MRI grading in the patients with early osteoarthritis

\begin{tabular}{|c|c|c|c|}
\hline \multirow{2}{*}{ MRI grading } & \multicolumn{2}{|c|}{ US grading } & \multirow{2}{*}{$\mathbf{n}, \%$} \\
\hline & 1 & 2 & \\
\hline 1 & 20 & 2 & $22(30.6 \%)$ \\
\hline 2 & 2 & 50 & $50(69.4 \%)$ \\
\hline $\mathrm{n}, \%$ & $\begin{array}{c}20 \\
(27.8 \%)\end{array}$ & $\begin{array}{c}52 \\
(72.2 \%)\end{array}$ & 72 \\
\hline Weighted Kappa (кw) & \multicolumn{3}{|c|}{0.933} \\
\hline $95 \% \mathrm{CI}$ & \multicolumn{3}{|c|}{0.841 to 1} \\
\hline
\end{tabular}

There was a highly statistically significant degree of agreement between US grading and MRI grading, $\kappa \mathrm{W}=0.858(95 \% \mathrm{CI}, 0.841$ to 1$), \mathrm{P}<0.001$.

\section{DISCUSSION}

The concepts regarding OA have dramatically changed in the past decade by development of the new imaging techniques as musculoskeletal ultrasound and MRI these techniques allow detection of early detection of pre-radiographic structural damages and not only the peri-articuralr bone but also in synovial membrane, ligaments, cartilage and menisci ${ }^{(1)}$.

Our study was carried on 50 patients with knee pain, They were 43 females and 7 males with mean age $29.5 \pm 4.4$ years old, Our results showed no significant statistical difference regarding age, sex, body mass index, smoking and occupation, This may be due-to selection of patients in young age (29.5), 86\% females, normal body mass index 23.5, 5\% smokers and 78\% with sedentary life.

Our study showed that patients group had higher WOMAC index and tenderness score than healthy group in this is in agreement with Levinger et al. ${ }^{(7)}$,they said that people with low level of vitamin $\mathrm{D}$ less than $50 \mathrm{nmol} / \mathrm{L}$ have poor knee function, greater knee pain and worse physical function compared with people with higher level of vitamin D.

Our study showed results according to WOMAC index and tenderness similar to Heidari et $\boldsymbol{a l}{ }^{(\mathbf{8})}$ which showed that status of serum vitamin $D$ levels in patients with knee OA compared to age matched group, They observed a positive association between serum vitamin D and knee OA in patients under 40 years old and noted a stronger association in younger participants.

The strong association between vitamin $\mathrm{D}$ deficiency and musculoskeletal pain which had been studied in knee and hip OA in correlation with low blood concentration of vitamin D and knee in Heidari et $\boldsymbol{a l} .{ }^{(\mathbf{8})}$ and Cao et $\boldsymbol{a l} .{ }^{(\mathbf{9})}$ they found a strong evidence for positive association between low level of vitamin D and cartilage loss, This is due-to the effect of vitamin $\mathrm{D}$ on cartilage, bone metabolism or both.

Our results showed that mean values of vitamin D level were $15.3 \pm 6.8 \mathrm{ng} / \mathrm{ml}$ which was statistically significant lower than control group,But there is no statistically significant difference regarding TSH level, We found that there is an association between vitamin D concentration, knee pain and knee OA these results are in agreement with Murat $\boldsymbol{e t} \boldsymbol{a l} .^{(\mathbf{1 0})}$ They said that there is association between vitamin D concentration and knee OA, Also Bergink et al..$^{(5)}$ showed a potential association between serum vitamin D levels and knee OA symptoms may occur through effect on cartilage metabolism, bone metabolism or both, also vitamin D are associated with higher prevalence of knee OA along with increased risk of disease progression. have suggested the potential benefits of vitamin D supplementation for knee OA, they showed significant associations between low vitamin D level and both prevalent knee joint space narrowing and progressive knee OA.

Our study results showed that patient group had a statistically significantly higher musculoskeletal ultrasound grading a, plain X-ray grading and MRI grading than control group, These results showed agreement with the systematic review performed by Cao et all ${ }^{(9)}$ which examined the associations between 
serum vitamin $\mathrm{D}$ and $\mathrm{OA}$, They found a strong evidence for an association between vitamin D and cartilage loss in knee joints, they also observed moderate evidence between low vitamin $\mathrm{D}$ and radiographic knee OA.

Our results showed the potent effect of musculoskeletal ultrasound in detecting early changes of knee OA which showed higher grading of musculoskeletal ultrasound in patient group than the controlled one, Our study Receiver operating characteristic (ROC) curve analysis revealed that serum vitamin $\mathrm{D}$ can fairly differentiate between patients with early OA from mild and moderate degeneration changes by ultrasound. The optimal sensitivity and specificity were $86 \%$ and $58 \%$, These results are in agreement with Riecke et al. (11) they showed the great role of musculoskeletal ultrasound in detecting the morphological changes of early knee OA, Our results in showed an agreement with Podlipska et al. ${ }^{(\mathbf{1 2})}$ they said that the musculoskeletal ultrasound is an accurate imaging method in detecting medial meniscus degeneration, medial femoral articular cartilage morphological degeneration, detection of femoral osteophytes and it has pre-radiographic detection of tissue structural OA degeneration

MRI and musculoskeletal ultrasound allow the early detection of pre-radiographic structural changes not only in the cartilage but also in the periarticular bone, synovial membrane,menisci and ligaments, Musculoskeletal ultrasound has a significant value in detection of synovial inflammation, effusion, decrease cartilage thickness and meniscial tears in patient without radiological signs of OA, MRI has the above advantages and also can detect bone marrow oedema and deep structures more that the musculoskeletal ultrasound ${ }^{(\mathbf{1})}$.

Our results showed a statistically significantly positive correlation between vitamin D level and femoral cartilage thickness in the Right lateral condylar(RLC) measurement and the Right inter-condylar (RIC) measurement in which patient with vitamin $\mathrm{D}$ deficiency has thinner femoral cartilage thickness in these measurement (RLC) and (RIC) than control group,so these results were in agreement with a study made by Malas $\boldsymbol{e t}$ al. ${ }^{(\mathbf{1 3})}$ which showed that there was a positive correlation between vitamin $\mathrm{D}$ level and musculoskeletal ultrasound measurements for a group with severe vitamin D deficiency, this study showed also that patient with vitamin $\mathrm{D}$ deficiency less than $10 \mathrm{nmol} / \mathrm{L}$ have thinner femoral cartilage thickness than patient with vitamin D level more than $10 \mathrm{nmol} / \mathrm{l}$, Our results showed an agreement to a study made by Okano et $\boldsymbol{a l} \mathbf{.}^{\mathbf{( 1 4 )}}$. They found that there is a strong correlation between radiographic medial tibio-femoral narrowing grade and the musculoskeletal ultrasound medial cartilage grade this prove that the musculoskeletal ultrasound was found to be a sensitive imaging technique for revealing cartilage damage and even minimal osteophytes.these results are in agreement with a study done by Randy $\boldsymbol{e t} \boldsymbol{a l} .{ }^{(15)}$ that showed moderate to strong correlation between MRI and musculoskeletal ultrasound measures of cartilage thickness in the medial femoral condyle.

\section{CONCLUSION}

Musculoskeletal Ultrasonography is a very important tool in detection of early OA. Vitamin D deficiency and insufficiency is correlated with thinning of femoral cartilage thickness and higher risk of early knee OA. Vitamin D Deficiency is correlated with musculoskeletal pain as the patient group has higher WOMAC index. Comparison between MRI and musculoskeletal ultrasound showed high concordance in the findings of cartilage changes of early OA patients.

\section{REFERENCES}

1. Favero M, Ramonda R, Goldring MB et al. (2015): Early knee osteoarthritis. Rheumatic and musculoskletal diseases Open, 1:e000062.

2. Michael JW, Schluter-Brust KU and Eysel $P$ (2010): The epidemiology, etiology, diagnosis and treatment of osteoarthritis of the knee. Dtsch Arztebl Int., 107(9): 152-162.

3. Royall NA, Farrin E, bahner DP et al. (2011): Ultrasound-assisted musculoskeletal procedures: A practical overvie of current literature. World J Orthop., 2(7): 57-66.

4. Vojtassak JR J and Vojtassak Sen J (2014): Ultrasound monitoring of the treatment of clinically significant knee osteoarthritis. europepmc.org/abstract/MED/24601702

5. Bergink AP, Zillikens MC, Van Leeuwen JPTM et al. (2016): 25-hydroxyvitamin D and osteoarthritis: A meta-analysis including new data. Semin. Arthritis. Rheum.,45(5): 539-546.

6. Hunter DJ, Arden N, Conaghan PG et al. (2011): Definition of osteoarthritis on MRI: Results of a Delphi exercise. Osteoarthritis Cartilage, 19: 963-9. 
7. PazitLevinger, RezaulBegg, Kerrie $M$ Sanders et al. (2017): The effect of vitamin D status on pain, lower limb strength and knee function during balance recovery in people with knee OA. Arch Osteoporosis, 12:83.

8. Heidari $B$, Heidari $P$ and Hajian-Tilaki $K$ (2011): Association between serum vitamin $D$ deficiency and knee osteoarthritis. International Orthopaedics, 35(11): 1627-1631.

9. Cao Y, Winzenberg T, Nguo K et al. (2013): Association between serum levels of 25hydroxyvitamin D and osteoarthritis: a systematic review. Rheumatology, 52(7): 1323-1334.

10. Murat C, Semih A, Haluk $C$ et al. (2018): Association between vitamin D concentratin and knee pain in patients with osteoarthritis. Peer J.,6:e4670.

11. Riecke BF, Christensen R, Torp-Pedersen $S$ et al. (2014): An ultrasound score for knee osteoarthritis: A cross-sectional validation study. Ostoearthritis and Cartilage, 22: 16751691.
12. Podlipska J, Guermazi A, Lehnkari P et al. (2018): Comparison of diagnostic performance of semi-quantitative knee ultrasound and knee radiograph with MRI. /www.nature.com / Scientific reports.

13. Malas FU, Kara M, Kaymak B et al. (2014): Ultrasonographic evaluation in symptomatic knee osteoarthritis: Clinical and radiological correlation. International Journal of Rheumatic Diseases, 17: 536-540.

14. Okano T, Tsugawa N, Masuda S et al. (2016): Regulatory activities of 2 beta-(3hydroxypropoxy)-1 alpha, 25-dihydroxyvitamin D3, a novel synthetic vitamin D3 derivative, on calcium metabolism. Biochem. Biophysical Res. Commun., 163: 1444-1449.

15. Randy J, Hsin-Min W, Daniel R, Robert A, Brian G (2017): Evaluation of knee cartilage thickness: A comparison between ultrasound and magnetic resonance imaging methods, Knee,24(2):217-223. 